\title{
A Glimpse of Expert Programmers' Mental Imagery
}

\author{
Marian Petre \\ Centre for Informatics Education Research \\ Faculty of Mathematics and Computing \\ Open University \\ Milton Keynes, U.K. \\ m.petre@open.ac.uk
}

\author{
Alan F. Blackwell \\ MRC Applied Psychology Unit \\ Cambridge, UK \\ alan.blackwell@mrc-apu.cam.ac.uk
}

KEYWORDS: mental representation, mental imagery, expert programmer behaviour.

\begin{abstract}
There is widespread anecdotal evidence that expert programmers make use of visual mental images when they are designing programs. In this study, expert programmers were directly questioned regarding the nature of their mental representations while they were engaged in a design task. This investigative technique was used with the explicit intention of eliciting introspective reports of mental imagery. The resulting transcripts displayed a considerable number of common elements. These suggest that software design shares many characteristics of more concrete design disciplines. They also provide promising areas for further investigation of software development support tools and design strategies.
\end{abstract}

\section{INTRODUCTION}

There is widespread anecdotal evidence that expert programmers build, in their minds, multidimensional structures which they manipulate or traverse in order to specify, predict, and simulate program behaviour. Researchers like Guindon, et al. (1987) make sweeping reference to these mental simulations: "The designer mentally explores and refines the mental model of the problem environment, especially through mental simulations." Similarly, mental simulation anecdotes litter Susan Lammers's interviews of well-known programmers (1986), e.g.:

"The first step in programming is imagining ... I like to imagine the structures that are being maintained, the structures that represent the reality $I$ want to code ... The code for the most part writes itself, but it's the data structures I maintain that are the key. They come first and I keep them in my mind throughout the entire process." (Charles Simonyi, page 15)

"You have to simulate in your mind how the program's going to work, and you have to have a complete grasp of how the various pieces of the program work together." (Bill Gates, page 73)

And similar anecdotes emerged in Petre's previous empirical study of expert programmers (1989):

"One of the earliest things is to visualize this structure in my head, a dynamic structure, so I can think about how things fit together and how they work ... and once I have the structure fairly strong and clear in my mind, I move it around and move around inside it, examining it and tweaking it ..." 
Each of these anecdotes includes two key elements: a structure of information, and how it works. These mental structures, part of initial solution planning, apparently embody information interrelationships and take into account manipulations and functions to be performed in processing information. They do not necessarily map onto data structures coded in programming languages - nor onto the 'objects' of recent popularity - yet they highlight the importance of structuring information, of making that structure apparent and accessible, and of making it amenable to required manipulations.

So, what is the nature of these mental structures? What might they reveal about how experts think about solutions and hence how might they inform our design of programming tools and representations? This paper presents a study which attempted to elicit mental imagery from expert programmers. That evidence is set in the context of the psychology literature on imagery.

\section{BACKGROUND TO THE STUDY:}

The study reported here followed from earlier research (Petre and Winder, 1988) in which expert programmers' descriptions of their problem solving were elicited as part of an investigation of how they matched programming languages to solutions. Many of those observed reported that they "start with the data structure", and so Petre (1989) investigated how experts derived data structures from problems. It became clear that experts' use of the term 'data structures' in the context of constructing abstract solution strategies doesn't necessarily correspond closely to 'data structures in code' - it encompasses much more information, such as what relationships exist, how the information will be manipulated, how the problem is being partitioned and structured, and so on. In experts' usage in this context, 'data structures' (as they conceived them) included considerable algorithmic information; the structure derived, not from the problem (although some data relationships are), but from the solution (in which the programmer interprets 'inherent' data relationships, imposes others, and organizes these to serve the strategy devised by the programmer). Moreover, the abstract data structures they described corresponded to a variety of code implementations, which in other contexts would be thought to be different data structures. For example:

"A specific resource has associated with it numerous characteristics (static) and an assignment vector (or list)(dynamic). Whether these are stored as properties of an atom, or as hash tables, or as components of a structure, or elements of a list, or entries in a vector ... is of indifference to me."

Therefore, it appears that the experts are not designing 'close to the code'; they are thinking abstractly and strategically, in some cases with a substantial translation to the implementation. (cf. Cox's (1996) argument that representations for problem solving should have sufficient expressive power for the task, but not more than is needed - but that "graphical tricks" can extend expressive power by allowing a single representation to stand for multiple models.)

So, what are these internal 'data structures', which are the currency of the experts' problem solving? Are there elements in common among experts, or other patterns of usage? How closely do these internal structures correspond to external representations, whether program code or visualisation tools? These are the questions which led to the study reported here, an attempt to elicit mental imagery used by expert programmers in designing solutions. 


\section{A SELECTIVE SUMMARY OF THE IMAGERY LITERATURE}

Individuals differ. There seems to be general acceptance in the modern literature that people form mental images. Individuals differ, in terms of their memory, transfer ability, cognitive preferences, repertoire of notational conventions, and so on. It appears that - just as perceptual skill can be trained (Petre, 1995) - imaging ability can develop, depending on exposure; for example, speakers of American sign language, which has a spatial component, display better imaging facility (Emmorey et al., 1993).

Problem solving and mental structure building. Although the authors have not discovered any reports on experimental investigations of the mental imagery used by programmers, the literaturc on expertise in problem solving does not contradict the familiar anecdotes. Observations on expert problem solving tend to be consistent across domains (for reviews, see Kaplan et al., 1986; Allwood, 1986) and, taken together, suggest a pattern of mental structure-building preliminary to efficient externalization of solutions. Experts tend to spend more time than novices in planning and evaluating. Experts are better able to form overviews, but thereafter they take longer to develop their understanding and representations, and they consider more fully interactions among functions or components of a system (Adelson et al., 1984). Experts form a detailed conceptual model of a system and tend to incorporate abstract entities rather than the concrete objects specific to the problem statement (Larkin, 1983). Their models accommodate multiple levels and are rich enough to support mental simulations (Jeffries et al., 1981; Adelson and Soloway, 1985). The indication, consistent with the anecdotes, is that experts spend some time building and exploring structures "in their heads" before making commitments to external representations.

Beyond the imagery debate: imagery involves different systems. The psychology literature on mental imagery has a chequered history, with considerable attention given to the great imagery debate (summarized in Kosslyn, 1994), concerning the extent of and level at which communication occurs between the various perceptual and cognitive subsystems. The crux of that debate is whether there is a uniform, underlying (propositional) encoding, or whether the only route between sub-systems is via externalization; Pylyshyn (1973) claimed that, if images are cognitively penetrable, then they are subject to propositional representation. That debate has been somewhat superceded by neurological and experimental evidence that imagery involves different systems (visual, spatial, verbal, temporal, propositional/semantic), which are usually handled in different parts of the brain. For example, Brooks (1968) used interference experiments to show that visualspatial and auditory-linguistic systems represent different processing modes; Farah et al. (1988) report that evidence has been accumulating for a dissociation between spatial and visual memory;

Imagery encompasses different modalities. There is varying evidence about imagery encompassing different modalities (sensory or cognitive modes or perceptual forms) . For example, Hitch \& Brandimonte (1995) concluded that "verbalization overshadows insight", that is, that requiring people to talk about things can over-ride insights through imagery. Finke, Pinker and Farah (1989) and Finke and Slayton (1988) argued that subjects' ability to re-interpret mental images suggests that they are not (or are not wholly) coded propositionally. Brooks $(1967,1968)$ and Byrne (1974) detected interference between perception and imagery, suggesting that an image may be an internally constructed perceptual description, using the same form in which external perceptions are coded. Logie et al. (1991) showed that image tasks are disrupted by intentional eye or arm movement tasks, suggesting that they are spatial rather than visual. Spatial and visual information seem to be orthogonal (Eysenck \& Keane, 1990; Bryant, 1992); the encoding of spatial information does not depend on the sensory channel of input. Melcher \& Schooler (1996) investigated interaction between modalities in their study of wine tasting, in which bad verbal encoders performed worse on recall tasks. 
Multiple mental representations. The literature gives reason to consider that we maintain multiple mental representations and that imagery is multiply-encoded. Kieras (1978) suggested that memory is propositional, but that it contains both semantic and perceptual information, i.e., incorporating information from different systems. Mani \& Johnson Laird (1982), in an investigation of mental representations of spatial descriptions, concluded that a propositional model was formed first, then a mental model using spatial layout, which if successful superceded the propositional model, i.e., serial representations. Payne (1993) suggested that long-term memory does not consist either of images or propositional representations, but the order of construction of the mental model, and that working memory contains a visuo-spatial image as well as scparate procedural information, i.e., multiple encoding. Many of the hypotheses about sources of insight are based on interactions between modalities. Chi, et al. (1989) showed that self-explainers perform better in problem solving; a self-explanation effect may operate during translation across modalities.

Imagery and insight. Various writers relate imagery to creative discovery or insight. Anderson \& Helstrup (1993) argue that mental imagery is a source of discovery and synthesis, while drawing is used in production and refinement of patterns. Kaufman (1980) hypothesized that visual imagery can be used for the organization of information needed in the initial, exploratory stage of problem solving, allowing simultaneous access to previously unrelated bits of information. Logie (1989) described an economy of images in memory, through which that access might be achicved: many informative elements are integrated together in a structural whole, increasing the available amount of information in working memory. Lindsay (1988) claimed that images differ from deductive propositional representations, in that they allow inferences that are not based on proof procedures. Goel (1995), although he was describing external representations, argued that dense, amorphous, ambiguous symbol systems (like sketching or visualisation) have an important place in cognition, and correlate with creative, explorative, ill-structured phases of problem solving. Finke, Pinker and Farah (1989) and Finke and Slayton (1988) suggested that reinterpretation of images is one potential source for creativity. Bartlett (1927) wrote that imagery leads into bypaths of discovery.

Caveat to the literature. The most pertinent shortcoming of the imagery literature with regard to illuminating the nature of expert programmers' mental imagery is the nature of the tasks employed. Most of the studies deal with particular, usually concrete or real-world images and simple tasks, e.g., visualizing a metaphor, visualizing a geometric solid and answering questions about it that require some manipulation; searching environments imagined from verbal descriptions (Franklin \& Tverski, 1990), syllogisms (Johnson-Laird, Sternberg, and others), etc. Hence their conclusions might not generalize to a realm in which the imagery concerns complex, abstract, imagined structures. This relates to the distinction Scott \& Baron-Cohen (1996) make between two functions of imagery: mental images of absent but real things, and mental images of absent and unreal things. Goel (1995) also argues that there is a principled distinction to be made between design and non-design problems (although he relates this to external representation). Therefore, there is a need for some insight into the imagery of the "absent and unreal".

\section{THE APPROACH}

The work reported here involved structured observational studies and interviews attempting to elicit mental imagery, not controlled laboratory experiments. The dangers of this technique are well-known, especially as reported in the review by Ericsson and Simon (1985). The main problems with data from verbal reports are the likelihood that cognitive processes of interest are not accessible to introspection, and the possibility that the experimenter might bias the response by asking only for certain types of report. The content of the reports must not be taken at face value either. Information in non-verbal modalities will be verbalised in different ways by different subjects - this is a particular problem in studies of imagery. Finally, many people when asked to 
describe their thoughts do not base their reports on memories of specific cognitive events, but theorise about cognitive processes in general.

Experts are well-known for rationalizing their practice 'on-the-fly'. As reported by Schooler, Ohlsson \& Brooks (1993), there is evidence that solving insight problems relies on essentially nonreportable processes, even that verbalisation interferes with some important thought processes. On the other hand, although subjective tests may be suspect, they have in some cases been shown to be reliably consistent, and to produce results just as good as those from more objective tests (Katz, 1983) There is some evidence that self-ratings do correlate with demonstrated ability (Ernest, 1977) and are stable in cases where they do.

The intention of this investigation was to provoke a rich response from experts, in order to elicit an impression of their mental imagery and to begin to look at whether there are common elements among the imageries of individuals. This study used a combination of observation and interview techniques which had, in previous studies, proven effective at eliciting rich qualitative data of this ilk. It adopted a loose protocol of questions which appeared in pilot interviews to elicit descriptions that both surprised and satisfied those questioned. The ability to engender surprise and satisfaction have, in previous studies, been effective criteria for elicitation questions. When a subject is surprised during an interview, it suggests a divergence from routine rehearsed response, whether in the form of a new expression, a new perspective, or a re-consideration. Surprise and satisfaction together can be an indication of insight: "Is that what I did? - that explains why..." or "Did I say that? - yes, that is the way I think about it!" The study relied on subjects whose reports of activity in earlier studies corresponded well to other evidence of their activity, such as notes and observed actions, i.e., it relied on subjects who appeared to be 'good self-reporters'.

\subsection{The experts}

The ten experts, from both industry and academia, and from several countries in Europe and North America, share the same general background: all have ten or more years of programming experience; all have experience with large-scale, real-world, real-time, data- and computationintensive problems; all are acknowledged by their peers as expert. Half are proficient with one or more declarative languages. All had participated in previous studies.

The coding language used was not of particular interest in this investigation, but, for the record, a variety of styles was exercised in the examples, using languages including APL, C, C+t, Hypercard, macro-assembler, common LISP, Miranda, Prolog, and SQL. Those with experience of declarative languages did not design significantly different solutions from those without.

\subsection{Problems}

Programmers were asked to design solutions to one of the following problems (also used in the previous data structures study), or to a problem of their choice. The experts were asked to imagine themselves free of coding restrictions, and they were not asked to implement the solutions as code.

Noughts and crosses player: The program acts as an interactive player of noughts and crosses.

Academic timetable maker: The program coordinates available resources (e.g., lecturers, equipment, rooms) with available times so as to maximize access to facilities, to avoid conflicts among related facilities, and to consider independence or interdependence of resources (e.g., course pre-requisites, mandatory courses). 
Lexicon for sub-anagram solver: The lexicon is organized so that the sub-anagram solver can find quickly all words in a given language which can be constructed from some or all of the letters in a given word or phrase.

Pinball path predictor: The path of a pinball is determined by the pinball machine architecture (including bumpers, flippers, etc.), the initial push given the ball, and the current state of the pinball machine. (Bonus question: Consider changes in data structures required if two balls could be in motion at the same time.)

The problems were chosen to evoke rich discussions by addressing classic issues in data representation and by admitting both standard and innovative treatments. "Noughts and crosses player' is a small but non-trivial problem with a familiar superficial structure. 'Academic timetable maker' is a classic data manipulation problem whose solution relies on resolving competing priorities applied to complex relationships among potentially substantial data. The 'sub-anagram solver' is another classic problem in which the task is limited and the data extensive, and which suggests a strong inter-dependence between data structure and algorithm. 'Pinball path predictor' requires a re-interpretation of a physical structure.

\subsection{Questions}

Depending on how much time was available, the programmers were questioned during the programming tasks (or during some tasks and after others). Prompting questions were keyed to the moments when (or the moments just after) the experts showed signs of internally-focussed thinking, such as:

- pauses in writing activity

- closing eyes

- staring 'into space'

- staring at blank paper (fixedly or with eye movement)

- gesturing in the air

Sometimes the questions interrupted the reverie, sometimes they followed, allowing completion of the thought episode. The prompts were general, e.g.,:

"What do you see?"

"What colour is it?"

"Does it move?"

"Give me instructions to see what you're seeing"

"What strikes you about what's there?"

"What's there that you can't see?"

"What can you hear?"

"Do you hear any words?"

"What's going on?"

"Where are you now?"

Prompts were chosen in a modality (a sensory mode or perceptual form) other than that of recent answers. The immediacy of the response was noted.

The initial answers were followed up with other probes, e.g.,:

"Is that what it's like, or is that what you see?"

"How much have you left out?"

"Are there bits you can't describe?"

"What was this [gesture]?" 
After the tasks were completed, the experts were questioned about their previous responses and about their imagery in general. All notes and other products were collected, and the sessions were recorded.

The analysis was data-driven. Notes and transcripts were examined for the imagery accounts they contained, and imagery descriptions were grouped in terms of common elements. Attention was given to the context in which the account was given. Particular attention was given to the spontaneity of the accounts (as reflected by the fluency and immediacy of description and by the amount of prompting required), to the programmer's satisfaction with the account, to any discrepancies, and to any indications of surprise.

\section{THE IMAGERY DESCRIBED}

The experts demonstrated a readiness to describe the form and contents of their thinking, although sometimes they found it difficult to articulate, and sometimes they were more adept at describing what their imagery was not ... What follows is a digest of the sorts of inagery described. The descriptions in italics are in the experts' own words; the quotations given here are all immediate responses from the elicitation during problem solving.

\subsection{Dancing symbols}

"... it moves in my head ... like dancing symbols ... I can see the strings [of symbols] assemble and transform, like luminous characters suspended behind my eyelids ..."

"I see lines of code ... text and variables ... changes of variables against time ..." One of the experts described textual imagery as "text with animation".

\subsection{Mental description/discussion}

"I'm just talking to myself..."

"In order to design something, you need to be able to describe it to yourself ..."

Most of the experts described a sort of verbal imagery, in which parts of the problem were described or 'discussed' mentally. Sometimes this verbal imagery accompanied other imagery; sometimes it occurred in isolation. Sometimes it took visual form (see 'dancing symbols' above), but more usually it did not.

\subsection{Auditory imagery}

"It buzzes ... there are things I know by the sounds, by the textures of sound or the

loudness ... it's like I hear the glitches, or I hear the bits that aren't worked out yet ..." All of the experts described sound as an element in their imagery, although most did not describe it as a typical element. Some described mental verbalizations, but not all of the auditory imagery was verbal; the experts also described auditory presentations of solution characteristics, with auditory qualities like loudness or tone reflecting some aspect of the solution, like level of activity or type of data.

\subsection{Visual imagery}

"values as graphs in the head ... flip into a different domain ... transform into a combined graph ... (value against time; amplitude against frequency; amplitude against time) ..." All of the experts described some visual imagery, and a variety of mental visualisations were described. Some were mental simulations, as described below. Others were described as "spaces of possibility", c.g., a look-up table for tcchniques, used in pre-solution reasoning when the expert 
was choosing a general strategy. Many of the visual images described bore some resemblance to standard external representations, although often these would be dymamic in the mind, changing with different dimensions, or augmented by other views or additional information.

\subsection{Machines in their minds}

\section{i. Abstract machines}

"slow-speed time ... movement of coefficients and button pushes ... a machine grovelling through it (munch munch)"

All of the experts described a sort of dynamic mental simulation, a 'machine in the mind'. Some of these were vivid, colourful, 'physical' structures - described by one expert as a "great, bristling, multi-coloured scaffolding of pipework and gadgets floating in space" - sometimes described as being in more than four dimensions. Others were logical structures, e.g., "The machines are like data structures - but as operated on ..." Often, data was visible as it flowed through the simulation. All of the experts described moving inside these active simulations, as though they (their perspective) moved around, inside, and through the image.

One expert described setting such a mental machine going to run 'independently':

"[imagery] is a way of harnessing the mental machine for tandem problem solving ... if there's a problem I can't solve ... I get the mental machine to solve a subset of the problem, and I need to see what it's doing in order to understand how that activity relates to the larger problem ..."

\section{ii. Pictures of implementations}

"object oriented ... nets of stuff ... poking inputs and see what filters through ... sucking:

I'm ready, send me another one..."

"It's a dynamic map ... this process talks to that process: this processor talks to that processor..."

Some of the mental simulations were described as augmented pictures of a 'physical machine' or an implementation (in a form such as a block diagram, a structure of objects, or a schematic) overlaid with data flow or process flow. (cf. claims by Finke, Pinker and Farah (1989) that images can be manipulated in a way that simulates transformations of physical objects, allowing successive states to be inspected.)

Many of the difficult programming problems - and those most difficult to reason about - invole the concatenation of tools or programs, e.g., outputting probe results into files (or pipes) to another tool, rather than just displaying results on screen. Hence, experts spend time modelling the behaviour of partially known objects, e.g., this computer must talk to that remote peripheral, which we don't know much about. It is for this sort of problem that the experts seem to use pictures of implementations.

\section{iii. Mechanical analogy}

These mental simulations were distinguished by the experts from mechanical analogies, which were discussed as a different (inferior) form of imagery:

"some people, in order to make things thinkable about, generate mechanical analogies -

e.g., two-scalar values represented as sliders"

The implication was that mechanical analogy was either constraining or potentially misleading.

\subsection{Surfaces}

Many of the experts described a strongly spatial, mathematically-oriented imagery of "solution surfaces' used in "prospecting around in the equation space" either to identify solutions or to 
identify simplifications that will lead to solutions. This imagery typically arises in connection with finding the correct solutions to a set of multi-dimensional, complex equations; it is described as a reduction of the multi-dimensional equation space into a two-dimensional surface where height or depth indicates 'goodness'.

"It's like describing all the dimensions of a problem in $2 D$, and in the third dimension you're putting closeness to a solution."

Seeking a solution in the space is imagined as traversing this surface, either to identify appropriate numeric values or to identify algebraic simplifications.

"It's like driving across a desert looking for a well. What you actually have is good solutions distributed across this desert like small deep wells and your optimizer trundling along looking for them..."

The surfaces were described variously in terms of wells (hence well-finding), attractors ("like heavy objects dropped in a rubber sheet"), or hills (hence hill-climbing), but the experts considered them equivalent. The surfaces in the imagery appear to vary among problems and classes of problems, so that experts described classes in terms of surface characteristics, e.g.: flat plains with evenly distributed wells, clustered wells of varying depths, wells with 'lips', prickly valleys with many local minima appearing as 'noise' obscuring a large depression, wells in clusters of known number, and so on.

\subsection{Landscapes}

All of the experts described a strongly spatial imagery, a surface or landscape of solution components over which they could 'fly', with different parts of the solution residing in different regions, and with a sense of different parts of the solution being visible even when distant in the landscape. Experts talked about knowing where things were in the landscape:

"... oh, that happens over there ... it's on the horizon, so I can keep an eye on it,but I don't really need to know..."

This landscape imagery was typically discussed in connection with large, multi-component solutions. There seems to be more variation in this type of imagery than was elicited in this study; the elicited descriptions had insufficient detail to enable us to elaborate this category.

\subsection{Presences}

“... no place holders, no pictures, no representation ... just the notion, the symbol entities, semantic entities and the linguistic token ... atomic notions. They just 'are"

All of the experts described a sort of imagery that was not verbal, visual, or physical. This was an imagery of presence (or knowledge) and relationship (sometimes location) which included qualities of the 'entities' present. These qualities conveyed the nature or character of the entity and were 'perceived' as a sort of notional (not tactile) pressure or texture, or as a tension or relative density between non-visual regions. Several experts described the qualities in terms of sensation, but then clarified that this did not correspond to external sensory experience. These images were subject to movement, change, or transformation. One expert described this imagery as "knowing in the dark". Another described it as "perturbations in non-visual space ... not always in 3D space or in sensual space". 


\section{COMMON ELEMENTS/CHARACTERISTICS}

Despite considerable individual variation, a number of common elements emerged in the experts' descriptions. Some elements were not used to the same extent; for example, most experts incorporate colour in their imagery some of the time, and a few usually do so, but some of them never do. The same was true of non-verbal sound. Other elements or characteristics were common, not just among the experts, but among their different sorts of imagery. Those are described here.

\subsection{Stoppably dynamic}

All of the images were described as dynamic, but subject to control, so that the rate could be varied, or the image could be frozen. In some cases, the activity could be reversed or the image restored to a previous state.

\subsection{Variable selection}

The 'resolution' of the imagery was not uniform; the experts chose where to put their attention at any given moment, and different regions of the imagery were described as coming in and out of focus. Information outside the focus might be undefined, or unsolved, or soluble, or solved; mainly, it was deemed not important at the moment.

"I don't need to think about that; it's enough to know it's over there ..."

(cf. Arnheim's (1969) discussion of "complete things perceived incompletely" in mental imagery as a positive quality, "the product of a selectively discerning mind", p. 105)

\subsection{Adjustable granularity of abstraction}

The experts described reasoning about aspects of solutions in more or less detail, depending on what interested them:

"... don't worry about what the detail is ... don't plow through each of the internal objects ..."

They chose what was general, and what particular. Some also described holding a simplified image in tandem with a more elaborate one, in order to consider a simpler analog to a knotty problem.

\subsection{Provisional, variable}

All of the imagery could accommodate incompleteness and provisionality, which were usually signalled in the imagery in some way, e.g., absence, fuzziness, partial shading, distance in a landscape, change of tone. (cf. Fishbein's (1987) argument that internal images have two characteristics which contribute to creative discovery: that they are not fully developed, and that they are associated with intrinsic feelings about their correctness; and Miller's claim (1993) that the vagueness of an image is critical to its utility.)

\subsection{Many dimensions}

All of the experts reported using more than four dimensions. The extra dimensions were usually associated with additional information (such as overlaid data flows or links to external representations), different views, or strategic alternatives.

"... small number of dimensional structures, $4+1$ say, (e.g., two sizes of time that are different enough to be different dimensions)..." 


\subsection{Multiplicity}

All of the experts described simultaneous, multiple imagery. Some alternatives existed as different regions (so that one alternated imagery by 'turning your head'). Some existed as overlaid or superimposed images, which became more or less transparent depending on the programmer's focus. Some existed as ready transformations or "morphing", e.g., "changes of scrutiny become textual changes", or as "projection onto translations". Some existed on different mental planes: "levels ... alternative representations, not connected together ..."

\section{7. 'The name business'}

Although some of the imagery is largely non-verbal, the experts all talked about the ready ability to label entities in the imagery. They clarified that not all imagery carried explicit labels (although some did encompass a 'name awareness'), but that the labels were available and accessible.

"The name business is absolutely necessary to understand things."

"Of course everything is labelled."

The relationship between imagery and explanation warrants further investigation.

\section{A CAUTIOUS CONCLUSION}

\subsection{On validity}

The cohort interviewed, although small, is arguably representative of expert programmers. But there is no assumption here that expert programmers are representative of any other population. The distillation is not claimed to cover all possible sorts of imagery, nor is the distribution of images taken to be representative. We do believe that, within the obvious limitations of the study, the distillation does give a valid glimpse of some of the mental imagery actually used by such people.

The criteria of surprise and satisfaction provide some basis for suggesting that the descriptions given are arguably close representations of the experts' mental imagery. The experts' surprise at their own accounts indicates at least that they were not just repeating practiced descriptions, that they were articulating things not usually articulated, and that they were impressed by the differences from their own expectations - their own rationalized versions- of what they imagine. Their satisfaction indicates at least that they accepted their accounts as plausible.

\subsection{Imagery for construction, not debugging:}

The imagery elicited here relates to solution construction. Many of the experts made comments suggesting that a different imagery may be employed during debugging:

"... the possibilities of debugging at bottom level from here are zero"

"In debugging, you only do it mentally for the difficult ones: intermittent, incomplete capture of the stimulus..."

(cf. Fox's (1914) conclusion that conflict in problem solving is favourable to use of imagery, while smooth thinking is not.)

\subsection{Imagery as a design technique}

There are a number of interesting characteristics of these imagery descriptions. The heavy reliance on non-verbal imagery supports cognitive and historical studies of design in several other disciplines. Goel (1995) describes a cognitive theory of architectural design that relies on imagelike representations. Ferguson (1992) presents historical arguments for the primacy of imagery in 
engineering design. Miller (1984) follows Dreistadt (1968) in describing the importance of imagery in scientific discovery. Winner and Casey (1992) argue on the basis of psychometric measures that fine art students display a relative superiority in image generation.

This study supports these cognitive theories of design from other disciplines. Programmers, like other designers, believe that much of design is non-verbal. And yet all of the experts maintained that verbal or symbolic labels were readily accessible, and that verbal description and discussion were typical parts of problem solving. The common elements that emerge from the considerable individual differences are striking: the multiplicity of modalities, stoppable dynamism, variable selection, provisionality and incompleteness, adjustable granularity, extra dimensions, simultaneous multiple images.

Of particular note is the distance of the imagery from programming languages and from programming paradigms. As phrased by one expert: "the advantage is that they [images] don't carry the baggage of the paradigm - and so might assist in finding things outside the paradigm." Both Denis (1991) and Cox (1996) argue that the main issue in problem solving is to identify the most appropriate visualisation for a given problem - and that some problems become trivial given a suitable representation. And yet the external representations - the programming notations - on which many programmers believe that they focus their problem solving are at some remove from the internal images adopted by experts in constructing solutions.

\subsection{Look next at tools}

One of the places to look next is at the sorts of tools experts build for themselves, which presumably complement and supplement their thinking, even if they don't mimic their internal representations. As argued in various places regarding various domains (e.g., Lansdown, 1993; Shenk, 1991; Fish and Scrivener, 1990), visualisation via sketching is part of the process of externalisation of concepts, an essential component of designing. It may be that the external visualizations which experts build for themselves are likewise part of the process of externalisation of concepts.

The things that one can visualise 'clearly' within one domain tend not to be what experts build their own tools for. Most errors that consume expert time are not within-paradigm, but looking below the paradigm at internal operation in order to debug abnormal behaviour of entities that are correct within the paradigm, i.e., unknown side-effects, rather than syntactic errors. So visualisations within-paradigm are uninformative. As a result, experts have a tendency to create a visualisation for a particular problem (e.g., specific data structure) even if it will never be useful for another problem. This sort of custom visualisation may well reveal something about how experts identify the most appropriate visualisation for a given problem.

\subsection{Conclusion}

Any investigation of what lies inside people's minds is problematic. Experimental psychology has led us to value only what can be demonstrated, but that paradigm has not provided sufficient insight into the realm of complex, abstract, imagined structures. For our early ventures into that realm, careful elicitation can provide valid insight. Strong commonalities emerged among the images described by the ten expert programmers studied, suggesting that there are accessible lessons to be elicited about how programmers construct solutions. The results presented here provide a glimpse of what experts do when they 'sit and stare.' 


\section{ACKNOWLEDGEMENTS}

The authors are grateful to the expert programmers without whom the paper would not be possible. Thomas Green and Peter Eastty each provided essential commentary at crucial moments.

Alan Blackwell's research is funded by a collaborative studentship from the Medical Research Council and Hitachi Europe Ltd. He is grateful to the Advanced Software Centre of Hitachi Europe for their support.

\section{REFERENCES}

Adelson, B., and Soloway, E. (1985) The role of domain experience in software design. IEEE Transactions on Software Engineering, SE-11(11), 1351-1360.

Adelson, B., Littman, D., Ehrlich, K., Black, J., and Soloway, E. (1984) Novice-expert differences in software design. In: Interact '84 : First IFIP Conference on Human-Computer Interaction. Elsevier.

Allwood, C.M. (1986) Novices on the computer: a review of the literature. International Journal of Man-Machine Studies, 25, 633-658.

Anderson, R.E. \& Helstrup, T. (1993) Visual discovery in mind and on paper. Memory and Cognition, 21(3), 283-293.

Arnheim, R. (1969) Visual Thinking. University of California Press.

Bartlett, F.C. (1927) The relevance of visual imagery to thinking. British Journal of Psychology, $18(1), 23-29$.

Brooks, L.R. (1968) Spatial and verbal components of the act of recall. Canadian Journal of Psychology, 22, 349-368.

Brooks, L.R. (1967) The suppression of visualization in reading. Quarterly Journal of Experimental Psychology, 19, 289-299.

Byrne, Brian (1974) Item concreteness vs. spatial organization as predictors of visual imagery. Memory and Cognition, 2 (1A), 53-59.

Chi, M.T.H., Bassok, M., Lewis, M.W., Reimann, P., and Glaser, R. (1989) Self explanations: how students study and use examples in learning to solve problems. Cognitive Science, 13, 145-182.

Cox, R. (1996) Analytical reasoning with multiple external representations. Unpublished doctoral dissertation. University of Edinburgh.

Denis, M. (1991) Imagery and thinking. In: C. Cornoldi \& M.A. McDaniel (eds.), Imagery and Cognition. New York: Springer-Verlag.

Dreistadt, R. (1968). An analysis of the use of analogies and metaphors in science. The Journal of Psychology, 68, 97-116

Emmorey, K., Kosslyn, S.M. \& Bellugi, U. (1993). Visual imagery and visual-spatial language: enhanced imagery abilities in deaf and hearing ASL signers. Cognition, 46, 139-181.

Ericsson, K.A. \& Simon, H.A. (1985). Protocol analysis: Verbal reports as data. Cambridge, MA: MIT Press.

Ernest, C.H. (1977) Imagery ability and cognition: a critical review. Journal of Mental Imagery, $1(2), 181-216$.

Farah, M.J. (1988) Is visual imagery really visual? Overlooked evidence from neuropsychology. Psychological Review, 95, 307-317.

Farah, M.J., Hammond, K.M., Levine, D.N., \& Calvanio, R. (1988) Visual and spatial mental imagery: dissociable systems of representation. Cognitive Psychology, 20, 439-462.

Ferguson, E.S. (1992) Engineering in the Mind's Eye. Cambridge, MA: MIT Press.

Finke, R.A., and Slayton, K. (1988) Explorations of creative visual synthesis in mental imagery. Memory and Cognition, 16, 252-257. 
Finke, R.A., Pinker, S., and Farah, M.J. (1989) Reinterpreting visual patterns in mental imagery. Cognitive Science, 13 (1), 51-78.

Fischbein, E. (1987) Intuition in Science and Mathematics: An Educational Approach. Dordrecht, Holland: Kluwer.

Fish, J., and Scrivener, S. (1990) Amplifying the mind's eye: sketching and visual cognition. Leonardo, 23 (1), 117-126.

Fox, C. (1914) The conditions which arouse mental images in thought. British Journal of Psychology, 6, 420-431.

Franklin, N. \& Tverski, B. (1990) Searching imagined environments. Journal of Experimental Psychology: General, 119 (1), 63-76.

Goel, V. (1995) Sketches of Thought. Cambridge, MA: MIT Press.

Guindon, R., Krasner, H., Curtis, B. (1987) Cognitive processes in software design: activities in early, upstream design. Interact '87. 383-388.

Helstrup, T., and Anderson, R.E. (1996) On the generality of mental construction in imagery: when bananas become smiles. European Journal of Cognitive Psychology, 8 (3), 275-294. abstract and concrete elements...different images, different memory usage

Hitch, G.J., Brandimonte, M.A., and Walker, P. (1995) Two types of representation in visual memory: evidence from the effects of stimulus contrast on image combination. Memory and Cognition, 23, 147-154.

Jeffries, R., Turner, A.A., Polson, P.G., and Atwood, M.E. (1981) The processes involved in designing software. In: J.R. Anderson (Ed.), Cognitive Skills and Their Acquisition. Erlbaum. 255-283.

Kaplan, S., Gruppen, L., Leventhal, L.M., and Board, F. (1986) The Components of Expertise: A Cross-Disciplinary Review. (The University of Michigan).

Katz, A.N. (1983) What does it mean to be a high imager? In: J.C. Yuille (ed.), Imagery, Memory and Cognition: Essays in Honor of Allan Paivio. Hillsdale, NJ: Erlbaum.

Kaufman (1979) Visual imagery and its relation to problem solving. Oslo, Norway: Universitetsforlaget.

Kieras, D. (1978) Beyond pictures and words: alternative information-processing models for imagery effects in verbal memory. Psychological Bulletin, 85 (3), 532-554.

Kosslyn, (1994) Image and the Brain: The Resolution of the Imagery Debate. Cambridge, MA: MIT Press.

Lammers, S. (1986) Programmers at Work. Redmond, Washington: Microsoft Press,

Lansdown, J. (1993) Visualising design ideas. In: Proceedings of BCS Conference on "Interacting with Images".

Larkin, J.H. (1983) The role of problem representation in physics. In: D. Gentner and A.L. Stevens (Eds.), Mental Models. Erlbaum.

Lindsay, R.K. (1988) Images and inference. Cognition, 29 (3), 229-250.

Logie, R.H. (1989) Characteristics of visual short-term memory. European Journal of Cognitivc Psychology, 1, 275-284.

Logie, R.H., and Marchetti, C. (1991) Visuo-spatial working memory: visual, spatial or central executive? In: R.H. Logie \& M. Denis (eds.), Mental Images in Human Cognition. Amsterdam: Elsevier. 105-115.

Mani, K., \& Johnson-Laird, P.N. (1982) The mental representations of spatial descriptions. Memory and Cognition, 10 (2), 181-187.

Melcher, J.M., \& Schooler, J.W. (1996) The misremembrance of wines past: verbal and perceptual expetise differentially mediate verbal overshadowing of taste memory. Journal of Memory and Language, 35 (2), 231-245.

Miller, A.I. (1984).Imagery in Scientific Thought: Creating 20th-Century Physics. Cambridge MA: MIT Press.

Miller, G.A. (1993) Images and models, similes and metaphors. In: A.Ortony (ed.), Metaphor and Thought, 2nd edition. Cambridge: Cambridge University Press, 357-400. 
Payne, S.J. (1993) Memory for mental models of spatial descriptions: an episodic-constructiontrace hypothesis. Memory and Cognition, 21 (5), 591-603.

Petre, M. (1989) Finding a Basis for Matching Programming Languages to Programming Tasks. Unpublished doctoral dissertation. University College London.

Petre, M. (1995) Why looking isn't always seeing: readership skills and graphical programming. Communications of the ACM, 38 (6), 33-44.

Petre, M., and Winder, R.L. (1988) Issues governing the suitability of programming languages for programming tasks. In: People and Computers IV: Proceedings of HCI'88. Cambridge University Press.

Pylyshyn, Z. (1973) What the mind's eye tells the mind's brain. Psychological Bulletin, 80, 1-24.

Schenk, P. (1991) The role of drawing in the graphic design process. Design Studies, 12 (3), 168181.

Schooler, J.W., Ohlsson, S., and Brooks, K. (1993) Thoughts behond words: when language overshadows insight. Journal of Experimental Psychology: General, 122 (2), 166-183.

Scott, F.J., \& Baron-Cohen, S. (1996) Imagining real and unreal things: evidence of a dissociation in autism. Journal of Cognitive Neuroscience, 8 (4), 371-382.

Sternberg, R.G. (1980) Representation and process in linear syllogistic reasoning. Journal of Experimental Psychology: General, 109, 119-159.

Winner, E. \& Casey, M.B. (1992). Cognitive profiles of artists. In G.C. Cupchik \& J. Lászlo (Eds), Emerging visions of the aesthetic process: Psychology, semiology and philosophy. Cambridge: Cambridge University Press 